\title{
Action Potential Modulation of Neural Spin Networks Suggests Possible Role of Spin
}

\author{
(Dated: February 23, 2004)
}

\author{
Huping $\mathrm{Hu}^{* 1} \&$ Maoxin $\mathrm{Wu}^{\dagger}$ \\ *Biophysics Consulting Group, 25 Lubber Street, Stony Brook, New York 11790, USA \\ ${ }^{\dagger}$ Department of Pathology, Mount Sinai School of Medicine, New York, New York 10029, USA
}

\begin{abstract}
In this paper we show that nuclear spin networks in neural membranes are modulated by action potentials through $J$-coupling, dipolar coupling and chemical shielding tensors and perturbed by microscopically strong and fluctuating internal magnetic fields produced largely by paramagnetic oxygen. We suggest that these spin networks could be involved in brain functions since said modulation inputs information carried by the neural spike trains into them, said perturbation activates various dynamics within them and the combination of the two likely produce stochastic resonance thus synchronizing said dynamics to the neural firings. Although quantum coherence is desirable and may indeed exist, it is not required for these spin networks to serve as the subatomic components for the conventional neural networks.
\end{abstract}

Tremendous progress has been made in neuroscience at cellular ${ }^{1}$, molecular $^{2}$ and atomic levels ${ }^{3}$. As an extension, we have been exploring whether certain subatomic events play a role in brain functions ${ }^{4}$. For instance, nuclear spins are basic quantum bits for encoding information and have long relaxation times after excitations ${ }^{5}$ and, on the other hand, neural membranes are saturated with spin-carrying nuclei.

Figure 1 shows the range of electric field strength $E_{m}$ inside the neural membranes during a typical action potential as calculated from $E_{m}=\frac{V_{m}}{d}$ where $V_{m}$ and $d$ are respectively the membrane voltage and thickness. It oscillates between -9 to +6 million volts per meter during the course of each action potential. These strengths are comparable to those causing electroporation of cell membranes and dielectric

\footnotetext{
${ }^{1}$ Correspondence Author: E-mail drhu@ quantumbrain.org or drhu@ att.net Voice/Fax 212-898-1103
} 
breakdown of many materials ${ }^{6}$ at which the covalent bonds of the constituent molecules are torn apart. So it significantly affects the conformations and collective dynamics of the neural membrane components such as phospholipids, cholesterols and proteins. Indeed, voltage-dependent ion channels perform their functions through electric field induced conformation changes of the constituent proteins ${ }^{3}$ and studies on the effects of electric fields on lipids support the above conclusion ${ }^{7,8}$.

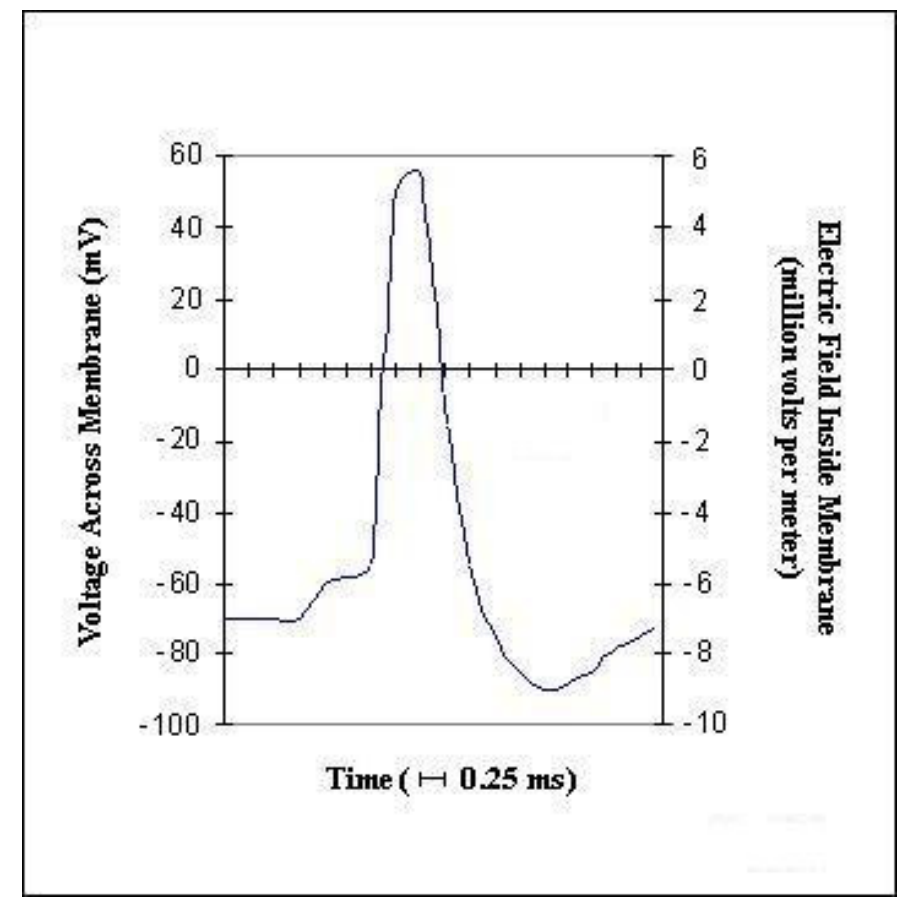

Figure 1. Electric field strength inside neural membrane during the course of an action potential. The calculation is down by assuming a typical membrane thickness of about $10 \mathrm{~nm}$ and the results are shown in the unit of one million volts per meter with "-" and "+" indicating that the direction of electric field is respectively pointing outward or inward inside the neural membrane.

The spins carried by the nuclei such as ${ }^{1} \mathrm{H},{ }^{13} \mathrm{C}$ and ${ }^{31} \mathrm{P}$ inside the neural membranes form complex intra- and inter-molecular spin networks through various intramolecular J- and dipolar couplings and both short- and long-range intermolecular dipolar couplings. Since J-coupling is the indirect interaction between two nuclear spins through covalent bonds and dipolar coupling is the direct interaction of two nuclear spins through space, their strengths and anisotropies strongly depend on the 
conformations of the neural membrane components ${ }^{9,10}$. Further, the chemical shielding of each nuclear spin also depends on the conformations of surrounding covalent bonds $^{11}$. Thus, when these spin networks are subjected to the enormous changing electric field produced during each action potential, the J-coupling, dipolar coupling and chemical shielding tensors oscillate with it, although nuclear spins themselves do not directly interact with electric fields. Studies on the effects of electric fields on these tensors ${ }^{9-11}$ also support this conclusion.

In the simple case of two $1 / 2$-spins inside neural membranes coupled to each other through isotropic J-coupling $J=J_{z z}=J_{x x}=J_{y y}=J_{R}+J_{A}$, the Hamiltonian of the system is $\hat{H}=h\left(J_{R}+J_{A}\right)\left(\hat{I}_{1 z} \hat{I}_{2 z}+\hat{I}_{1 x} \hat{I}_{2 x}+\hat{I}_{1 y} \hat{I}_{2 y}\right)$ where $J_{R}$ is the J-coupling at resting potential and $J_{A}$ is the first-order contribution to $J$ from action potential modulation thus it is a function of membrane voltage $V_{m}$. For a given value of $V_{m}$ the two $1 / 2$-spins form a triplet consisting of $|1\rangle=|\uparrow \uparrow\rangle,|3\rangle=\frac{1}{\sqrt{2}}(|\uparrow \downarrow\rangle+|\downarrow \uparrow\rangle)$ and $|4\rangle=|\downarrow \downarrow\rangle$ and a singlet $\left.|2\rangle=\frac{1}{\sqrt{2}}(\uparrow \downarrow\rangle-|\downarrow \uparrow\rangle\right)$ with energies $E_{1}=E_{3}=E_{4}=\frac{1}{4} h\left(J_{R}+J_{A}\right), E_{2}=-\frac{3}{4} h\left(J_{R}+J_{A}\right)$

thus an energy gap $J=h\left(J_{R}+J_{A}\right)$. The J-coupling strengths between ${ }^{1} \mathrm{H}$ and ${ }^{1} \mathrm{H}$ are typically in the range of 5-25 Hz. Further, J-couplings among biologically available nuclear spins such as ${ }^{1} \mathrm{H},{ }^{13} \mathrm{C}$, and ${ }^{31} \mathrm{P}$ are in the range of $5-250 \mathrm{~Hz}$ that are also the frequency spectra of various brain activities associated with different functional states. The possible significance of this fascinating fact will be considered elsewhere.

In the principal axes system of dipolar coupling tensor $\boldsymbol{D}$ for the two $1 / 2$-spins, $\hat{H}=h\left(J_{R}+J_{A}+D_{R}+D_{A}\right) \hat{I}_{1 z} \hat{I}_{2 z}+h\left(J_{R}+J_{A}-\frac{1}{2} D_{R}-\frac{1}{2} D_{A}\right)\left(\hat{I}_{1 x} \hat{I}_{2 x}+\hat{I}_{1 y} \hat{I}_{2 y}\right)$ is the Hamiltonian with both isotropic J-coupling $J=J_{R}+J_{A}$ and dipolar coupling $D=D_{z z}=-\frac{1}{2} D_{x x}=-\frac{1}{2} D_{y y}=D_{R}+D_{A}$ where $D_{R}$ is the dipolar coupling at resting potential and $D_{A}$ is the first-order contribution to $D$ from action potential modulation thus it is also a function of membrane voltage $V_{m} . D$ is typically in the range of $100 \mathrm{~Hz}$ 
to $10 \mathrm{kHz}$. It can be verified that $|1\rangle,|3\rangle|4\rangle$ and $|2\rangle$ are also the eigenstates of the above Hamiltonian with energies $E_{1}=E_{4}=\frac{1}{4} h\left(J_{R}+J_{A}+D_{R}+D_{A}\right), E_{3}=\frac{1}{4} h\left(J_{R}+J_{A}\right)-$ $\frac{1}{2} h\left(D_{R}+D_{A}\right)$ and $E_{2}=-\frac{3}{4} h\left(J_{R}+J_{A}\right)$. Thus, dipolar coupling has no effect on the singlet state but partially removes the energy degeneracy of the triplet states thus producing zero-field splitting.

Further, the chemical shielding tensor $\sigma$ of each nuclear spin also contains contribution from action potential modulation of its surrounding covalent bonds. That is, for the first $1 / 2$-spin $\sigma_{1}=\sigma_{1 R}+\sigma_{1 A}$ and for the second $1 / 2$-spin $\sigma_{2}=\sigma_{2 R}+\sigma_{2 A}$ where $\sigma_{1 R}$ and $\sigma_{2 R}$ are the chemical shielding tensors at resting potential and, $\sigma_{1 R}$ and $\sigma_{2 R}$ are the first-order contribution to $\sigma_{1}$ and $\sigma_{2}$ respectively from action potential modulations thus they are functions of membrane voltage $V_{m}$. So when the effects of both internal and external magnetic fields $\mathbf{B}_{i}$ and $\mathbf{B}_{e}$ are taken into accounts the total Hamiltonian for the two $1 / 2$-spin system in neural membranes is $\hat{H}=-\hbar \gamma_{1} \hat{\mathbf{I}}_{1} \cdot\left(1-\sigma_{1 R}-\sigma_{1 A}\right) \cdot\left(\mathbf{B}_{1 i}+\mathbf{B}_{1 e}\right)-\hbar \gamma_{2} \hat{\mathbf{I}}_{2} \cdot\left(1-\sigma_{2 R}-\sigma_{2 A}\right) \cdot\left(\mathbf{B}_{2 i}+\mathbf{B}_{2 e}\right)+$ $h \hat{\mathbf{I}}_{1} \cdot\left(\mathbf{J}_{R}+\mathbf{J}_{A}\right) \cdot \hat{\mathbf{I}}_{2}+h \hat{\mathbf{I}}_{1} \cdot\left(\mathbf{D}_{R}+\mathbf{D}_{A}\right) \cdot \hat{\mathbf{I}}_{2}$ where $\mathbf{B}_{1 i}, \mathbf{B}_{1 e}, \mathbf{B}_{2 i}$ and $\mathbf{B}_{2 e}$ are respectively the internal and external magnetic fields at the locations of first and second $1 / 2$-spins without chemical shielding and, $\gamma_{1}$ and $\gamma_{2}$ are respectively the gyromagnetic ratios of the said first and second 1/2- spins. In general, microscopically $\frac{\left|\mathbf{B}_{i}\right|}{\left|\mathbf{B}_{e}\right|} \gg>1$ at each spin location as shown later but macroscopically $\left\langle\mathbf{B}_{i}\right\rangle_{\mathbf{r}}=0,\left\langle\mathbf{B}_{e}\right\rangle_{\mathbf{r}} \neq 0,\left\langle\mathbf{B}_{i}\right\rangle_{t}=0$ and $\left\langle\mathbf{B}_{e}\right\rangle_{t} \neq 0$ where $\mathbf{r}$ and $t$ respectively denote spatial and time average. So in many cases the effects of $\mathbf{B}_{e}$ on these spin networks are small.

These results from consideration of a simple two-1/2-spin system in neural membranes demonstrate that the large neural spin networks inside the membranes can form complex modulated structures through action potential driven oscillations of $J$ coupling, dipolar coupling and chemical shielding tensors. Thus, the neural spike trains 
of various frequencies can directly input information carried by them into these spin networks.

The fluctuating internal magnetic fields are produced by the paramagnetic species such as $\mathrm{O}_{2}$ and $\mathrm{NO}$ and spin-carrying nuclei themselves such as ${ }^{1} \mathrm{H},{ }^{13} \mathrm{C}$ and ${ }^{31} \mathrm{P}$. Table 1 shows the maximal magnetic field strengths produced by the magnetic dipoles of the unpaired electrons of $\mathrm{O}_{2}$ and $\mathrm{NO}$ and the nucleus of ${ }^{1} \mathrm{H}$ along the axes of said dipoles at given distances. Because the magnetic dipole moment of an unpaired electron is 658 times larger than that of the ${ }^{1} \mathrm{H}$ nucleus, $\mathrm{O}_{2}$ and $\mathrm{NO}$ can respectively produce magnetic fields 1,316 and 658 times larger than ${ }^{1} \mathrm{H}$. As distance $r$ increases, the strength of the magnetic dipole field quickly attenuate according to $B=\frac{\mu_{0} m}{4 \pi r^{3}}$ where $\mu_{0}$ is the permeability of free space and $m$ is the magnetic dipole moment. In addition, $\mathrm{O}_{2}$ and NO are hydrophobic small molecules so their concentrations in neural membranes are much higher than in aqueous solutions such as cytoplasma ${ }^{12}$. As they rapidly tumble and diffuse, they produce microscopically strong and fluctuating magnetic fields. Indeed, $\mathrm{O}_{2}$ are the predominant sources of internal magnetic fields in neural membranes as evidenced by the strong effect of $\mathrm{O}_{2}$ on spin-spin and spin-lattice relaxation rates ${ }^{12,13}$.

\begin{tabular}{|l|l|l|l|}
\hline \multicolumn{4}{|c|}{ Table 1. Magnetic Fields Produced by $\mathrm{O}_{2}, \mathrm{NO}$ and ${ }^{1} \mathrm{H}$} \\
\hline Distance $(\AA)$ & $\mathrm{O}_{2}$ (Tesla) & NO (Tesla) & ${ }^{1} \mathrm{H}$ (Tesla) \\
\hline 1.0 & 3.713940 & 1.856970 & 0.002821 \\
\hline 2.0 & 0.464243 & 0.232122 & 0.000353 \\
\hline 3.0 & 0.137553 & 0.068777 & 0.000104 \\
\hline 4.0 & 0.058030 & 0.029015 & 0.000044 \\
\hline 5.0 & 0.029712 & 0.014856 & 0.000023 \\
\hline 10.0 & 0.003714 & 0.001857 & 0.0000003 \\
\hline
\end{tabular}


These fluctuating internal magnetic fields continuously perturb the neural spin networks. The intensities of said perturbations depend on the concentrations of $\mathrm{O}_{2}$ and NO that are highly regulated in the brain. Thus, these perturbations not only activate various modulated dynamics within the neural spin networks but also are likely capable of enhancing the synchronization of these dynamics to the neural spike trains through non-linear processes such as stochastic resonance that is known to occur in the brain ${ }^{14}$ 15 . So, stochastic resonance of dipolar splitting transitions and spin-forbidden singlettriplet transitions are possible inside the neural membranes under said modulations and perturbations. Stochastic resonance in two-state nuclear spin system was demonstrated by NMR spectroscopy ${ }^{16}$.

It is therefore suggested that the collective dynamics of the neural spin networks under modulations by action potentials and perturbations by fluctuating internal magnetic fields represent meaningful information to the brain. An analogy to this suggestion is the mechanism of liquid crystal display (LCD) where information-carrying electric voltages applied to the pixel cells change the optical properties of the constituent molecules such that when lights pass through these cells their phases get rotated differently which in turn represent different information to the viewer of the LCD screen ${ }^{17}$. According to this suggestion, significant ${ }^{1} \mathrm{H}$ replacements by ${ }^{2} \mathrm{H}$ and large external disturbances of the collective dynamics of the neural spin networks will affect the functional states of the brain to certain extent. Further, drug-induced large changes to membrane structures and $\mathrm{O}_{2}$ pathways in neural membranes have similar adverse effects. These predications are testable and provide alternative interpretations to the causes of neural effects produced by some drugs and external stimulations. For example, the effect of transcranial magnetic stimulations (TMS) on cognitive functions ${ }^{18}$ can be partly attributed to the direct disturbances of the dynamics of the said spin networks by TMS and the cause of unconsciousness by general anaesthetics can be 
explained as the direct consequence of their effects on neural membrane structures and $\mathrm{O}_{2}$ pathways inside ${ }^{19}$.

However, how can we explain based on the above suggestion that cognitive functions seem in general insensitive to environmental and even medical strength external magnetic fields such as those generated by the power lines and the ones used in MRI? First, the strengths of environmental magnetic fields are in the range of $10^{-4}-10^{-6}$ Tesla $^{20}$, For example, the magnetic field strength of the earth is about $5 \times 10^{-5}$ Tesla. In comparison, the internal fluctuating magnetic fields can be as high as several Tesla as indicated by Table 1 . Thus, the microscopically strong and fluctuating internal magnetic fields overshadow them. But the strengths of magnetic fields used in clinical and research MRI systems are in the range of 0.064 to $8.0 \mathrm{Tesla}^{21}$ that is comparable to or even higher than the strengths of said internal magnetic fields. So, additional explanations are called for. Indeed, the net magnetization of nuclear spins even by magnetic field of several Tesla is only about a few ppm at room temperature ${ }^{5}$ which shows that even strong static magnetic fields only have small effects on the thermal dynamics of the neural spin networks. Third, to the extent that said spin networks are disturbed by external magnetic fields, it is argued that most of these disturbances do not represent meaningful information to the brain and, further, the brain likely have developed other mechanisms through evolution to counter the effects of external magnetic fields. In the cases where external magnetic disturbances were reported to have observable effects on cognition, the above suggestion provides a basis for interpreting these effects as said disturbances contain meaningful information to the brain.

Although quantum coherence is not required for the neural spin networks to serve as the subatomic components for the conventional neural network according to the above suggestion, it likely exist within some parts of said networks as recent studies in 
other fields suggest ${ }^{4}$. For example, when nematic liquid crystal is irradiated with multifrequency pulse magnetic fields in room temperature, the ${ }^{1} \mathrm{H}$ spins in its constituent molecules can form long-lived intra-molecular quantum coherence with entanglement for information storage ${ }^{22}$ and long-lived $(\sim .05 \mathrm{~ms})$ entanglement of two macroscopic spin ensembles in room temperature has also been achieved ${ }^{23}$. In this regard, there are quite a few quantum theories related to cognition ${ }^{24,25}$ but most of them either lack connectivity to known neural activities or suffer from the seemingly insurmountable but hotly debated decoherence effects ${ }^{26,27}$. We emphasize here that spin is a fundamental quantum process and reveals itself through the structure of the relativistic quantum equation for fermions and is connected to the structure of space-time ${ }^{28}$. Indeed, spin was shown to be responsible for the quantum effects ${ }^{29,30}$. Thus, when exploring whether quantum effects are involved in brain functions, we have considered spin as a likely candidate ${ }^{4}$.

In conclusion, we have shown in this report how neural spin networks are modulated by action potentials and perturbed by microscopically strong and fluctuating internal magnetic fields and suggested that these combined influences could produce various dynamics within said spin networks that represent meaningful information to the brain. We cautiously add here that the nuclear spins inside neural membranes could be the fundamental cognitive pixels. Our results implicate the possibilities of spin-based artificial mind and medicine and provide insights into the workings of general anesthetics and the mechanisms of reported neural effects of various magnetic fields.

\section{REFERENCES}

1. Marder, E., Abbott, L. F., Turrigiano, G. G., Liu, Z. \& Golowasch, J. Memory from the dynamics of intrinsic membrane currents. Proc. Natl. Acad. Sci. USA. 93, 1348113486 (1996).

2. Hunt, S. P. \& Mantyh, P. W. The Molecular dynamics of pain control. Nature Rev. Neurosci. 2, 83-91 (2001). 
3. Morais-Cabral, J. H., Zhou, Y. \& MacKinnon, R. Energy optimisation of ion conduction rate by the K selectivity filter. Nature 414, 37-42 (2001).

4. Hu, H. P., \& Wu, M. X. Spin-Mediated Consciousness Theory. arXiv e-print quant$\mathrm{ph} / 0208068$ (2002).

5. Gershenfeld, N. \& Chuang, I. L. Bulk spin resonance quantum computation. Science 275, 350-356 (1997).

6. Barnet, A. \& Weaver, J. C. Electroporation: a unified, quantitative theory of reversible electrical breakdown and mechanical rupture in artificial planar bilayer membranes. Bioelectrochem. Bioenerg.. 25, 163-182 (1991).

7. Sargent, D. F. Voltage jump/capacitance relaxation studies of bilayer structure and dynamics. J. Membr. Biol. 23, 227-247 (1975).

8. Saux, A. L., Ruysschaert, J. M. \& Goormaghtigh, E. Membrane molecule reorientation in an electric field recorded by attenuated total reflection Fouriertransform infrared spectroscopy. Biophys. J. 80, 324-330-125 (2001).

9. Grayson, M. Electric field effects on ${ }^{2} \mathrm{~J}_{\mathrm{HH}}$ spin-spin coupling constants. Int'l J. Mol. Sci. 4, 218-230 (2003).

10. Peshkovsky, A. \& McDermott, A. E. Dipolar interactions in molecules aligned by strong AC electric fields. J. Magn. Reson. 147, 104-109 (2000).

11. Buckingham, A. D. Chemical shifts in the nuclear magnetic resonance spectra of molecules containing polar groups. Can. J. Chem. 38, 300-307 (1960).

12. Marsh, D. Polarity and permeation profiles in lipid membranes. Proc. Natl. Acad. Sci. USA. 98, 7777-7782 (2001).

13. Prosser, R. S., Luchette, P. A., Weterman, P. W., Rozek, A. \& Hancock, R. E. W. Determination of membrane immersion depth with $\mathrm{O}_{2}$ : A high-pressure ${ }^{19} \mathrm{~F}$ NMR study. Biophys. J. 80, 1406-1416 (2001).

14. Bezrukov, S. M. \& Vodyanoy, I. Noise-induced enhancement of signal transduction across voltage-dependent ion channels. Nature 378, 362-364 (1995).

15. Simonotto, E., Riani, M., Seife, C., Roberts, M., Twitty, J. \& Moss, F. Visual perception of stochastic resonance. Phys. Rev. Lett. 78, 1186-1189 (1997).

16. Viola, L., Fortunato, E. M., Lloyd, S., Tseng, C. H. \& Cory, D. G. Stochastic resonance and nonlinear response using NMR spectroscopy. Phys. Rev. Lett. 84, 54665469 (2000).

17. Bryan-Brown, G. P., Brown, C. V., Sage, I. C. \& Hui, V. C. Voltage-dependent anchoring of a nematic liquid crystal on a grating surface. Nature 392, 365-367 (1998).

18. Walsh, V. \& Cowey, A. Transcranial magnetic stimulation and cognitive neuroscience. Nature Rev. Neurosci. 1, 73-79 (2000).

19. Hu, H. P., \& Wu, M. X. Mechanism of Anesthetic Action: Oxygen pathway perturbation hypothesis. Med. Hypotheses 57, 619-627 (2001). 
20. Marino, A. A. Environmental electromagnetic fields and public health. In Foundations of Modern Bioelectricity Marino, A. A., ed. (Marcel Dekker, New York, 1988).

21. Shellock, F. G. Magnetic Resonance Safety Update 2002: Implants and Devices. J. Magn. Resonan. Imaging 16, 485-496 (2002).

22. Khitrin, A. K., Ermakov, V. L. \& Fung, B. M. Information storage using a cluster of dipolar-coupled spins. Chem. Phys. Lett. 360, 161-166 (2002).

23. Julsgaard, B., Kozhekin, A. \& Polzik, E. S. Experimentally long-lived entanglement of two macroscopic objects. Nature 413, 400-403 (2001).

24. Donald, M. J. Quantum theory and the brain. Proc. R. Soc. A 427, 43-93 (1990).

25. Hameroff, S. \& Penrose, R. Conscious events as orchestrated spacetime selections. J. Conscious. Stud. 3, 36-53 (1996).

26. Tegmark, M. The importance of quantum decoherence in brain processes. Phys. Rev. E 61, 4194-4206 (2000).

27. Hagan, S, Hameroff, S. R. \& Tuszynski, J. A. Quantum computation in brain microtubules: Decoherence and biological feasibility. Phys. Rev. E 65, 061901 (2002).

28. Penrose, R. A spinor approach to general relativity. Ann. Phys. 10, 171-201 (1960). 29. Hestenes, D. Quantum mechanics from self-interaction. Found. Phys. 15, 63-78 (1983).

30. Salesi, G. \& Recami, E. Hydrodynamics of spinning particles. Phys. Rev. A 57, 98105 (1998). 